Originalien

Unfallchirurg $2021 \cdot 124: 366-372$

https://doi.org/10.1007/s00113-020-00866-8

Angenommen: 11. August 2020

Online publiziert: 15 . September 2020

๑) Der/die Autor(en) 2020

\section{Redaktion}

W. Mutschler, München

H. Polzer, München

B. Ockert, München
Martin C. Jordan' - Sebastian Jovic ${ }^{1}$ Fabian Gilbert - Andreas Kunz ${ }^{2}$. Maximilian Ertl ${ }^{3} \cdot$ Ute Strobl $^{4} \cdot$ Rafael G. Jakubietz ${ }^{1} \cdot$ Michael G. Jakubietz . Rainer H. Meffert ${ }^{1} \cdot$ Konrad F. Fuchs $^{1}$

'Klinik und Poliklinik für Unfall-, Hand-, Plastische und Wiederherstellungschirurgie, Universitätsklinikum Würzburg, Würzburg, Deutschland

${ }^{2}$ Institut für Diagnostische und Interventionelle Radiologie, Universitätsklinikum Würzburg, Würzburg, Deutschland

${ }^{3}$ Servicezentrum Medizin-Informatik (SMI), Universitätsklinikum Würzburg, Würzburg, Deutschland

${ }^{4}$ Verwaltung, Referat 3.3.2 - Medizincontrolling, Universitätsklinikum Würzburg, Würzburg, Deutschland

\title{
Qualitätssteigerung der Abrechnungsprüfung durch Smartphone-basierte Fotodokumentation in der Unfall-, Hand- und plastischen Chirurgie
}

\section{Einleitung}

Eine gut nachvollziehbare Dokumentation über Verletzungen, durchgeführte Operationen, vorliegende Haupt- und Nebendiagnosen sowie die Wundheilung während des stationären Aufenthalts ist für die Abrechnung zwischen Kliniken und Kostenträgern essenziell. Neben dem schriftlichen Befund und der radiologischen Bildgebung ist die digitale Fotodokumentation von Verletzungen und Wunden mittlerweile ein zentraler Bestandteil der Patientenakte. Derzeit ist unklar, welchen Stellenwert die digitale Fotodokumentation bei der Abrechnungsprüfung mit dem Kostenträger hat. Deshalb ist das Ziel dieser Studie, den Zusammenhang zwischen der Abrechnungsprüfung und einer Smartphone-basierten Fotodokumentation zu analysieren. Die Arbeitshypothese lautet, dass die systematische Fotodokumentation einen relevanten Einfluss auf die Dokumentation des klinischen Verlaufs hat und somit die korrekte Kodierung und Kostenabrechnung unterstützt. Dies kann dem Leistungserbringer bei Rechnungsprüfung mit dem Kostenträger helfen, den Erlös komplexer chirurgischer Fälle realistisch zu belegen.

\section{Material und Methoden}

\section{Smartphone-basierte Fotodoku- mentation}

Im Rahmen eines von der Landesregierung finanzierten Digitalisierungsprojekts am Universitätsklinikum Würzburg erfolgte zunächst die eingeschränkte Ausgabe von Tabletcomputern (iPad Generation 3-4; Fa. Apple, Cupertino, Kalifornien, USA) an ärztliches und pflegerisches Personal, um mit einer eigens konzipierten App auf Patientendaten im Kliniknetzwerk zugreifen zu können (UKW-Mobile App, SMI, Würzburg; - Abb. 1). Diese App erlaubt den Zugriff auf alle Patientendokumente wie z.B. Arztbriefe, Untersuchungsbefunde oder auf die radiologische Diagnostik. 2016 wurde diese Applikation um die Möglichkeit einer fallverknüpften Fotodokumentation erweitert. Hierbei werden die gewonnenen Fotos direkt mit Notizen im Picture Archiving and Communication System (PACS, Fa. Phö- nix PACS GmbH) gespeichert und sind somit ebenfalls jederzeit dezentral und sicher abrufbar. Durch das Digitalisierungszentrum für Präzisions- und Telemedizin (DZ.PTM) erfolgte ab 2018 u. a. die schrittweise Ausgabe von iPhones XR (Fa. Apple) an ärztliche Mitarbeiter zur Ausweitung der Fotodokumentation und besseren Implementierung der App in den klinischen Alltag. Im Jahr 2019 wurden alle Mitarbeiter der Klinik und Poliklinik für Unfall-, Hand-, Plastische und Wiederherstellungschirurgie aufgefordert, die App zur Fotodokumentation in der Notaufnahme, im OP und im Schockraum zu verwenden. Verletzungen, Ausgangsbefunde, Dekubiti, Wunden, Wundheilungsstörungen oder Infektionen wurden isoliert abgebildet und in das System eingespeist. Mit dieser Technologie ergibt sich die Möglichkeit, eine qualitativ hochwertige, systematische Fotodokumentation bei entsprechender Indikation vorzunehmen. 


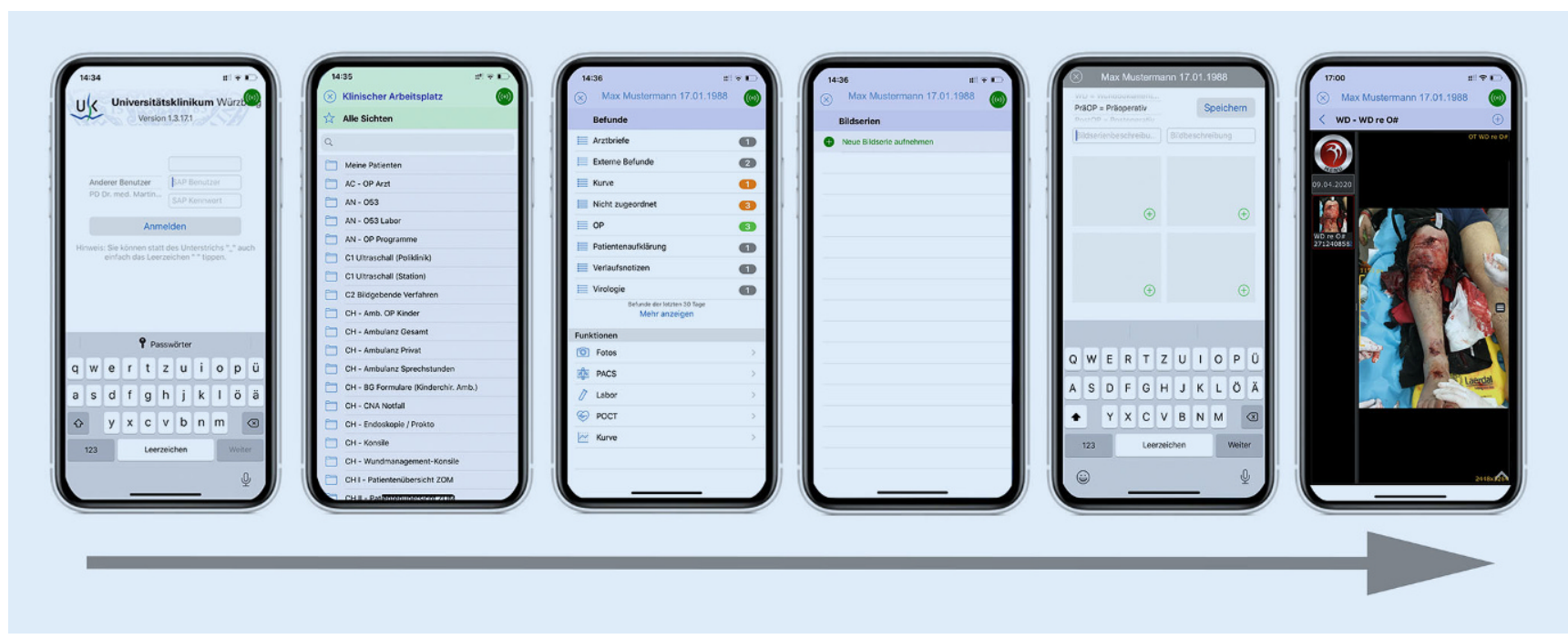

Abb. 1 ॥ Mittels UKW-Mobile App kann in wenigen Sekunden eine Fotodokumentation durchgeführt werden. In der passwortgeschützten App wird der Fall aufgerufen und das angefertigte Bild direkt im Archivierungsprogramm hinterlegt

\section{Datenschutz}

Die Berücksichtigung sämtlicher datenschutzrechtlicher Aspekte bei der Arbeit mit Patientendaten stellt im Alltag eine zwingende Voraussetzung dar. Die UKW-Mobile App ist eine Entwicklung der klinikeigenen IT-Abteilung und berücksichtigt die Datenschutz-Grundverordnung (DSGVO). Fotodokumente werden nach Auswahl des betroffenen Patienten innerhalb der App erstellt und nicht auf dem Gerätespeicher abgelegt. Somit wird verhindert, dass Dokumente unbeabsichtigt in nichtklinischen CloudSystemen hinterlegt werden. Ein Upload erfolgt ausschließlich auf das klinikeigene Serversystem. Wenn ein Patient auf einem Foto eindeutig zu erkennen ist, muss vor einer Verbreitung dieser Bilder eine Einwilligung eingeholt werden (Vorträge, Publikationen). In Fällen, in denen eine Zuordnung z.B. aufgrund einer sehr individuellen Verletzung möglich scheint, empfiehlt es sich ebenfalls, eine Einwilligung einzuholen. Es sollte immer berücksichtigt werden, dass die Fotodokumentation einer hilfsbedürftigen Person ohne medizinisches Ansinnen strafbar ist.

\section{Analyse der Abrechnungsprüfung}

Mithilfe des Medizincontrollings, des DRG-Beauftragten und den DRG-Assistenten erfolgte eine retrospektive Aus- wertung der MDK-Begehungen in der Unfall-, Hand- und plastischen Chirurgie der Monate Januar bis Dezember 2019. Grunddokument der Auswertung waren die vorliegenden Besprechungsprotokolle, aus denen die Argumentation und Begründung aller behandelter Fälle hervorgeht. Die anschließende Auswertung der Daten erfolgte pseudonymisiert. Ein Ethikvotum war dementsprechend nicht erforderlich. Von allen veröffentlichen Bildern liegt die unterschriebene Einwilligung betroffener Personen vor. Der klinische Verlauf der diskutierten Fälle wurde analysiert. Fälle, in denen eine Fotodokumentation eine OPS oder Diagnose sichern konnte, wurden herausgegriffen und die Veränderung der Abrechnung dokumentiert. Hierbei wurde zwischen einem erlösmindernden, einem erlössteigernden oder einem gleichbleibenden Effekt unterschieden. Der monetäre Gegenwert der erhaltenen oder verlorenen Diagnose bzw. Prozedur wurde erhoben und abschließend summiert (Internationale statistische Klassifikation der Krankheiten und verwandter Gesundheitsprobleme, 10. Revision, German Modification, Version 2019 mit Aktualisierung vom 01.11.2019 [ICD-GM-10 2019] sowie Operationenund Prozedurenschlüssel, Version 2019 mit Aktualisierungen bis zum 03.12.2018 [OPS-Version 2019], beide DIMDI sowie ID Diacos; ID Information und Doku- mentation im Gesundheitswesen $\mathrm{GmbH}$ \& Co. KGaA).

\section{Ergebnisse}

Im Jahr 2019 erfolgten 372 Fallbesprechungen zwischen dem Universitätsklinikum Würzburg und dem Medizinischen Dienst der Krankenkassen (MDK). Hierbei handelte es sich nur um Patienten aus der Unfall-, Hand- und plastischen Chirurgie. Von 372 Fällen lag bei 134 (36\%) eine Fotodokumentation im System vor. In $27(7,2 \%)$ Fällen half die Fotodokumentation zur Sicherung/Rechtfertigung/Verteidigung entweder einer OPS $(n=5 ; 1,3 \%)$, einer Hauptdiagnose $(n=10 ; 2,7 \%)$ oder Nebendiagnose $(n=3 ; 0,8 \%)$ sowie der Krankenhausverweildauer $(n=9 ; 2,4 \%)$. Pro oben genanntem fotodokumentierten Fall ergab sich eine durchschnittliche Erlössteigerung von $2119 €$. Die Summe aller Fälle erzeugte eine Erlössteigerung von 57.228€. Je verhandeltem Fall wurde noch eine Aufwandspauschale von $300 €$ addiert, womit sich abschließend eine Gesamtsumme von $65.328 €$ für das Jahr 2019 ergab (• Tab. 1).

\section{Diskussion}

Insgesamt ist die Datenlage zu Abrechnungsprüfungen durch den MDK in Kliniken spärlich. Unstrittig ist aber, dass durch die Abrechnungsprüfung 
Unfallchirurg 2021 124:366-372 https://doi.org/10.1007/s00113-020-00866-8

(c) Der/die Autor(en) 2020

M. C. Jordan · S. Jovic · F. Gilbert · A. Kunz · M. Ertl · U. Strobl · R. G. Jakubietz • M. G. Jakubietz · R. H. Meffert · K. F. Fuchs

Qualitätssteigerung der Abrechnungsprüfung durch Smartphone-basierte Fotodokumentation in der Unfall-, Hand- und plastischen Chirurgie

\section{Zusammenfassung}

Hintergrund. Die Fotodokumentation von offenen Frakturen, Wunden, Dekubitalulzera, Tumoren oder Infektionen ist ein wichtiger Bestandteil der digitalen Patientenakte. Bisher ist unklar, welchen Stellenwert diese Fotodokumentation bei der Abrechnungsprüfung durch den Medizinischen Dienst der Krankenkassen (MDK) hat.

Fragestellung. Kann eine Smartphone-basierte Fotodokumentation die Verteidigung von erlösrelevanten Diagnosen und Prozeduren sowie der Verweildauer verbessern? Material und Methoden. Ausstattung der Mitarbeiter mit digitalen Endgeräten (Smartphone/Tablet) in den Bereichen Notaufnahme, Schockraum, OP, Sprechstunden sowie auf den Stationen. Retrospektive Auswertung der Abrechnungsprüfung im Jahr 2019 und Identifikation aller Fallbesprechungen, in denen die Fotodokumentation eine Erlösveränderung bewirkt hat.

Ergebnisse. Von insgesamt 372 Fallbesprechungen half die Fotodokumentation in 27 Fällen (7,2\%) zur Bestätigung eines Operationen- und Prozedurenschlüssels (OPS) $(n=5 ; 1,3 \%)$, einer Hauptdiagnose $(n=10 ; 2,7 \%)$, einer Nebendiagnose $(n=3$; $0,8 \%)$ oder der Krankenhausverweildauer $(n=9 ; 2,4 \%)$. Pro oben genanntem Fall mit Fotodokumentation ergab sich eine durchschnittliche Erlössteigerung von $2119 €$. Inklusive Aufwandpauschale für die Verhand- lungen wurde somit ein Gesamtbetrag von $65.328 €$ verteidigt.

Diskussion. Der Einsatz einer Smartphonebasierten Fotodokumentation kann die Qualität der Dokumentation verbessern und Erlöseinbußen bei der Abrechnungsprüfung verhindern. Die Implementierung digitaler Endgeräte mit entsprechender Software ist ein wichtiger Teil des digitalen Strukturwandels in Kliniken.

Schlüsselwörter

Digitalisierung · Gesundheits-App · Künstliche Intelligenz · Plattform · Strukturwandel

\section{Smartphone-based photographic wound documentation improves the quality of medical accounting in orthopedic and plastic surgery}

Abstract

Background. Photographic documentation of wounds, decubitus ulcers, tumors, open fractures and infections is an important part of digital patient files. It is unclear whether the photographic documentation has an effect on medical accounting with health insurance companies.

Objective. It was hypothesized that Smartphone-based systematic photographic documentation can improve the confirmation of proceeds-relevant diagnoses and procedures as well as the duration.

Material and methods. Staff in the emergency room, operating theater, outpatient clinic and on the wards were equipped with digital devices (Smartphone, tablet) including a photo-app. Medical accounting with the health insurance companies and identification of all case conferences in which the photographic documentation had effected a change in proceeds were analyzed for 2019 in a retrospective manner.

Results. Overall, 372 cases were discussed of which 27 cases were affected by the digital photographic documentation. Photographic documentation was used for clarification of the operative procedure $(n=5)$, primary diagnosis $(n=10)$, secondary diagnosis $(n=3)$, and length of hospitalization $(n=9)$. An average of $2119 €$ was negotiated and added per case affected by photographic documentation. Hereby, a level 1 trauma center gained an estimated $65,328 €$ in revenue.

Discussion. The use of Smartphone based photographic documentation can improve the overall quality of patient files and thus avoid loss of revenue. The implementation of digital devices with corresponding software is an important component of the digital structural change in hospitals.

Keywords

Artificial intelligence · Database · Digital transformation · Photo app · Surgery relevante Erlöskürzungen durch lückenhafte Dokumentation möglich sind. So hat 2018 der MDK Nordrhein $17 \%$ aller Krankenhausfälle geprüft und in 50,3\% der Fälle eine Kürzung von durchschnittlich $2000 €$ pro Fall vorgenommen. Nur in $1 \%$ der Fälle ergab sich eine Korrektur zugunsten der Krankenhäuser. Gegenstände der Prüfung waren in 57,8\% die stationäre Verweildauer und in 30,6\% die Kodierung [1]. Dieser Sachverhalt bestätigt die hohe Wertigkeit einer präzisen Dokumentation, wenn möglich durch die Verwendung klinischer Fotos.
Im Vorfeld der Rechnungsprüfung werden üblicherweise fallspezifische Fragen der Krankenkassen durch den MDK an das Krankenhaus übermittelt. Neben den üblichen Dokumenten wie Ambulanzund Entlassbrief, OP-Bericht, Laborund Röntgenbefund können so im Vorfeld auch hilfreiche Fotodokumente aus dem Archiv bereitgestellt werden. Die hier vorgestellten Ergebnisse zeigen, dass eine Fotodokumentation insbesondere zur Bestätigung der Hauptdiagnose und zur Rechtfertigung der Verweildauer vorteilhaft ist. Es zeigt sich aber auch, dass die Fotodokumentation nur in relativ wenigen Fällen (7,2\%) einen Beitrag leisten kann. Auch wenn der Einsatz der Fotodokumentation ausgeweitet werden sollte, so bleibt diese Technik nur ein Baustein in der komplexen Abrechnungsprüfung. Eine gute schriftliche Dokumentation ist weiterhin unverzichtbar. Auffallend war in unserer Analyse, dass die Fotodokumentation überproportional häufig in der Hand- und Plastischen Chirurgie zur Klärung beitragen kann. Gerade in diesem Fachbereich hat die präoperative Fotodokumentation bei 
Tab. 1 Zusammenfassung aller Fallbesprechungen, in denen die Fotodokumentation einen erlösrelevanten Einfluss hatte. Als Änderungsgrund der

Abrechnung wird unterschieden zwischen OP-Prozedur (OPS), Haupt- und Nebendiagnose sowie der klinischen Verweildauer. Es handelt sich um ge-

schätzte Beträge entsprechend der Abrechnungstabelle zum Zeitpunkt der Untersuchung

\begin{tabular}{|c|c|c|c|c|}
\hline Fall & Diagnose & Fotodokumentation & MDK & Erlös \\
\hline 1 & $\begin{array}{l}\text { Quetschwunde, Hand } \\
\text { Durchtrennung GNB } 6\end{array}$ & Risswunde, D III, beugeseitig & Verweildauer & $671 €$ \\
\hline 2 & Risswunde, Oberarm & Großflächige Wunde & Hauptdiagnose & $2658 €$ \\
\hline 3 & $\begin{array}{l}\text { Implantatinfektion } \\
\text { Paraplegie }\end{array}$ & Wunden am Fuß & $\begin{array}{l}\text { Nebendiagnose } \\
\text { (Dekubitus) }\end{array}$ & $\begin{array}{l}\text { Keine Ände- } \\
\text { rung }\end{array}$ \\
\hline 4 & Schnittverletzung, Unterarm & Verzögerte Wundheilung & Verweildauer & $671 €$ \\
\hline 5 & Amputation, Daumen & $\begin{array}{l}\text { Amputat (Nachweis einer vollständigen } \\
\text { Amputation) }\end{array}$ & Hauptdiagnose & $8217 €$ \\
\hline 6 & $\begin{array}{l}\text { Schnittverletzung, Hand mit Durchtrennung von } \\
\text { A. und N. ulnaris und FCU }\end{array}$ & Schnittverletzung, Unterarm & Hauptdiagnose & $1380 €$ \\
\hline 7 & Pertrochantäre Femurfraktur & Dekubitus & $\begin{array}{l}\text { Nebendiagnose } \\
\text { (Dekubitus) }\end{array}$ & Keine Abzüge \\
\hline 8 & Dekubitus & Wundheilungsstörung & Verweildauer & $709 €$ \\
\hline 9 & Schnittwunde am Fuß & Risswunde & Verweildauer & $1260 €$ \\
\hline 10 & Offene Unterschenkelfraktur & III ${ }^{\circ}$ offene Fraktur & Hauptdiagnose & Keine Abzüge \\
\hline 11 & Bursitis olecrani & Infizierte Bursa & Hauptdiagnose & Keine Abzüge \\
\hline 12 & Fettschürze, Abdomen & Befunddokumentation & Hauptdiagnose & $3366 €$ \\
\hline 13 & Risswunde, Handrücken mit Strecksehnenverletzung & Risswunden am Handrücken & Verweildauer & Keine Abzüge \\
\hline 14 & Abdominoplastik & Fettschürze & Verweildauer & $2065 €$ \\
\hline 15 & $\begin{array}{l}\text { Unterschenkelamputation mit Wundheilungsstö- } \\
\text { rung }\end{array}$ & Nekrose am Stumpf & Verweildauer & $729 €$ \\
\hline 16 & Amputation, D II-V & Amputat (Nachweis der Replantation) & OPS & $9129 €$ \\
\hline 17 & Infiziertes Atherom & Infektfokus & OPS & $\begin{array}{l}\text { Keine Ände- } \\
\text { rung }\end{array}$ \\
\hline 18 & Dermatochalasis & Präoperative Weichteildokumentation & Verweildauer & $424 €$ \\
\hline 19 & Dekubitus & $\begin{array}{l}\text { Offene Wunde, glutäal } \\
\text { (Débridement }>4 \mathrm{~cm}^{2} \text { ) }\end{array}$ & OPS & $2826 €$ \\
\hline 20 & Periprothetische Femurfraktur & Dekubitus & Nebendiagnose & Keine Abzüge \\
\hline 21 & Pseudarthrose, Unterschenkel & Spalthautdeckung & OPS & Keine Abzüge \\
\hline 22 & Wundheilungsstörung, Unterschenkel & Defektausmaß & Hauptdiagnose & $1760 €$ \\
\hline 23 & Adipositas, geplanter „body lift“ & Fettschürze & Hauptdiagnose & $806 €$ \\
\hline 24 & Mittelhandamputation & Stumpf (Nachweis der Amputation) & Hauptdiagnose & $7441 €$ \\
\hline 25 & Verschleppter Infekt am Fuß nach Stichverletzung & $\begin{array}{l}\text { Aufnahmebefund mit Stichverletzung } \\
\text { und septischem Fuß }\end{array}$ & Hauptdiagnose & $7201 €$ \\
\hline 26 & Schnittverletzung, Hohlhand & Unfallbilder & Verweildauer & $3025 €$ \\
\hline 27 & $\begin{array}{l}\text { Osteomyelitis, Unterschenkel, behandelt mit freiem } \\
\text { Lappen }\end{array}$ & Defektgröße & OPS & $2890 €$ \\
\hline \multicolumn{4}{|c|}{ Zwischensumme } & $57.228 €$ \\
\hline \multicolumn{4}{|c|}{ Aufwandpauschale je Fall (300€) } & $8100 €$ \\
\hline \multicolumn{4}{|c|}{ Gesamtbetrag } & $65.328 €$ \\
\hline GNB & erven-Bündel, FCU M. flexor carpi ulnaris & & & \\
\hline
\end{tabular}

körperformenden Eingriffen eine lange Historie. Außerdem sollte der klinische Verlauf gut dokumentiert werden, um die Notwendigkeit weiterer Eingriffe oder der stationären Behandlung $\mathrm{zu}$ belegen. Insbesondere Weichgewebsveränderungen wie Wunden, Schwellungen und Infektionen sowie deren Verläufe können nicht durch Röntgendiagnostik oder Laborwerte nachvollzogen werden, weshalb die Fotodokumentation eine sinnvolle Ergänzung darstellt (• Abb. 2 und 3). Unabhängig davon ist die Fotodokumentation möglicherweise bei juristischer Aufarbeitung nützlich [2].
Vorteile der Smartphone-basierten Fotodokumentation mittels App sind, im Gegensatz zum Einsatz einer Digitalkamera, die einfachere Handhabung sowie die direkte Zuordnung zur Patientenakte. In der Vergangenheit war die Fotodokumentation aufgrund fehlender Geräte häufig nicht möglich [3]. Zudem 

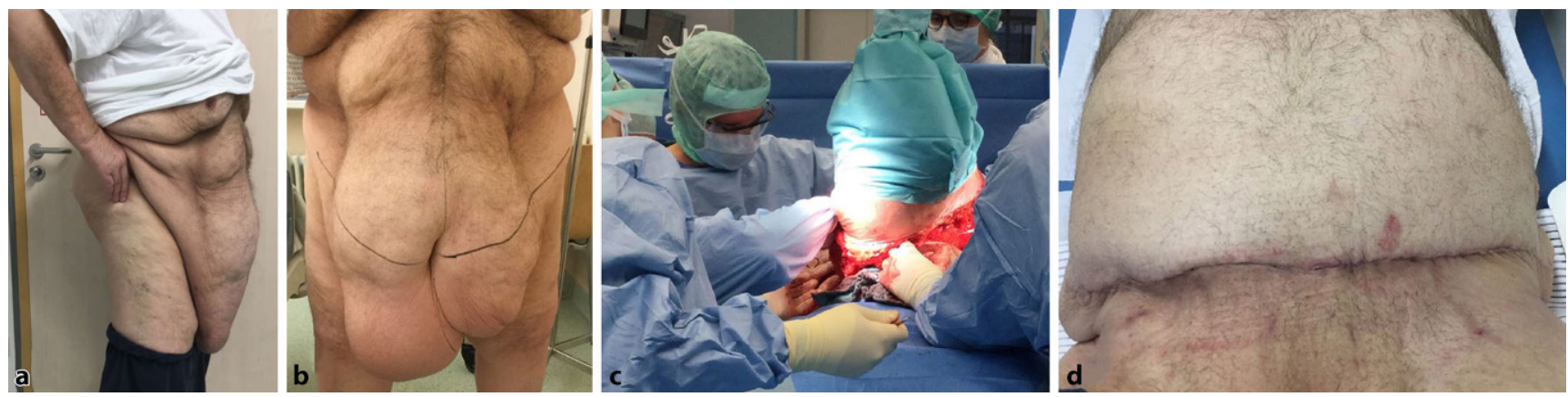

Abb. 2 ム Fotodokumentation des Ausgangsbefunds sowie der Operationsplanung bei massiver Fettschürze (a, b; Fall 23). Das Ausmaß des Eingriffs mit großen Resektionsflächen und entsprechendem Risiko für Komplikationen wird hieraus ersichtlich (c). Die Wundheilung kann durch die regelmäßige Fotodokumentation adäquat nachvollzogen werden (d)
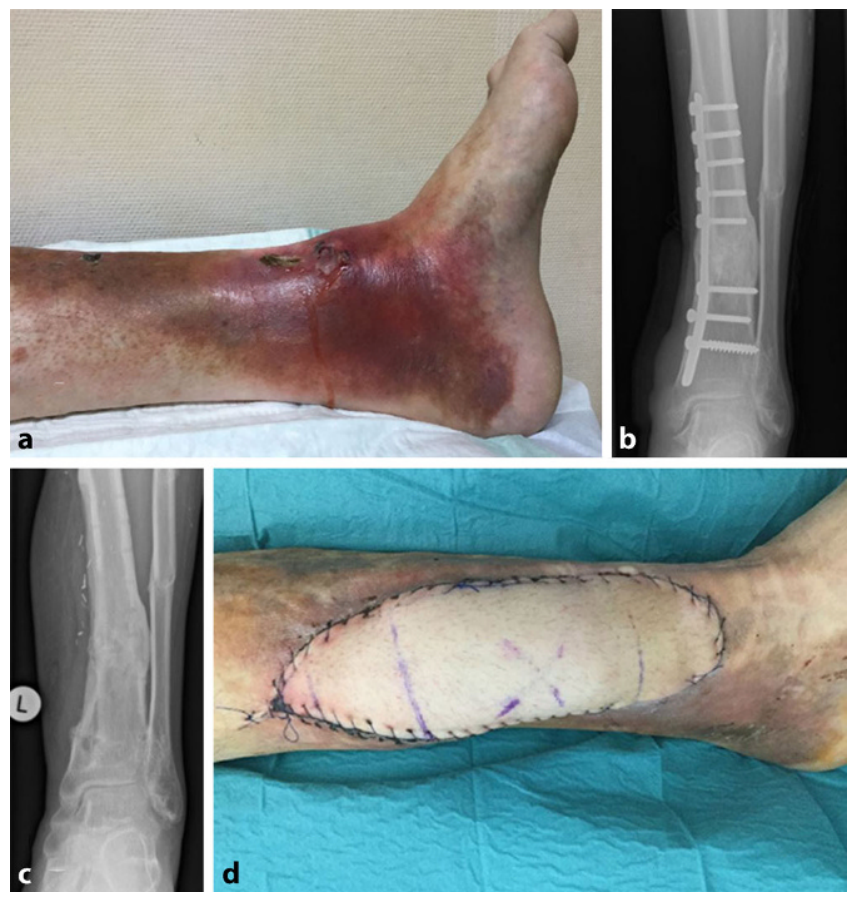

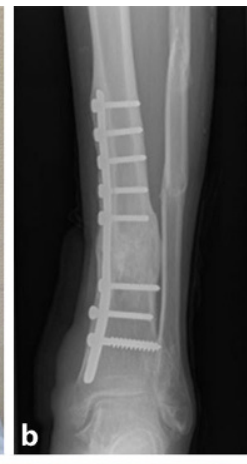

Abb. $3<$ Dokumentation einer chronischen Osteomyelitis (a, b; Fall Nr. 27), welche mit Débridement, Metallentfernung und Lappenplastik behandelt wurde. Defektgröße und Lappenindikation werden hiermit belegt $(c, d)$ gab es bei der Archivierung und Qualität der Fotos immer wieder Probleme. Wichtig ist eine konstante Qualität der Fotodokumentation, welche in Zukunft durch eine standardisierte Verfahrensanweisung weiter gesteigert werden kann $[4,5]$. Hierfür ist die Entwicklung einer „standard operating procedure“ (SOP) mit definierten technischen Kriterien denkbar, wie z. B. gleichbleibendem Abstand und Winkel zum Objekt sowie einheitlicher Beleuchtung und abgebildeter Größenreferenz. Eine solche SOP wäre die Grundlage für die Anwendung einer automatisierten Auswertung (künstliche Intelligenz) und könnte die Abrechnung durch automatisierte Aus- wertung der Fotodokumentation weiter verbessern [6].

Ein weiterer Vorteil der systematischen Fotodokumentation ist die Verwendung der Bilder in der Ausbildung und Lehre. Außerdem kann die Bearbeitung von hausinternen oder sogar überregionalen Konsilanforderungen vereinfacht werden [7], insbesondere dann, wenn die Fotodokumentation delegierbar und eine persönliche Konsultation nicht erforderlich ist. Die Mitbeurteilung durch erfahrenere Kollegen oder andere Fachdisziplinen kann unter deutlicher Zeitersparnis erfolgen. Im Vergleich zur umständlichen und zeitraubenden Fotodokumentation mittels Digitalkamera ist die Benutzerfreundlichkeit („ease of use") ein Vorteil, welcher zu mehr Akzeptanz und Einsatz führt. Keinesfalls darf der Austausch von Patientenbildern über ungeschützte Messenger-Dienste oder E-Mail-Verkehr erfolgen.

Die Fotodokumentation offener Frakturen wird mittlerweile von nationalen und internationalen Fachgesellschaften dringend empfohlen [8]. Somit sollen eine unnötige mehrfache Abnahme des Verbands verhindert sowie die bessere Planung operativer Prozeduren ermöglicht werden. Des Weiteren kann die Fotodokumentation im Rahmen von Gutachten oder der Feststellung von Dauerschäden eine hilfreiche Ergänzung sein [9].

Ein Nachteil der am Universitätsklinikum Würzburg durchgeführten Art der Fotodokumentation sind die Anschaffungskosten der mobilen Endgeräte, der Software und der Unterhaltungskosten. Die Vorteile der Smartphone-Nutzung wie z.B. Steigerung der Effizienz und Arbeitserleichterung gehen aber über die Fotodokumentation hinaus und sind Teil eines notwendigen digitalen Strukturwandels. So werden die Smartphones auch zur innerklinischen Kommunikation verwendet. Die Kosten für eine solche Infrastruktur sollten deshalb bei anstehenden Investitionsverhandlungen berücksichtigt werden, da diese in Zukunft auch von qualifizierten Arbeitnehmern eingefordert werden [10, 11].

Limitationen unserer Studie ergeben sich aus der retrospektiven Datenanalyse, welche das Risiko einer Fehlinterpretation birgt. Ein prospektives Studiendesign mit Protokoll, definierten Einschluss- 
kriterien, einer Vergleichsgruppe mit Randomisierung und festgelegten Outcome-Parametern kann die Aussagekraft zukünftiger Studien deutlich verbessern. Ein Kritikpunkt bezüglich der Smartphone-Nutzung im Krankenhaus ist abschließend erwähnenswert. Erkenntnisse aus der privaten Smartphone Anwendung deuten auf psychosoziale Risiken dieser Technologie hin, und der Einsatz am Arbeitsplatz ist umstritten [12]. Permanente Ablenkung durch die Smartphone-Nutzung während wichtiger Besprechungen, Beschäftigung mit dem Smartphone anstatt mit dem $\mathrm{Pa}$ tienten während der Visite oder kommentarlose Fotodokumentation ohne den expliziten Hinweis darauf, dass es sich nicht um ein privates Gerät handelt, können nach eigener Beobachtung zu Konflikten mit Patienten oder Kollegen führen.

\section{Fazit für die Praxis}

\section{- Die Smartphone-basierte Abbildung von Wunden oder intraoperativen Befunden kann die Qualität der Dokumentation verbessern. \\ - Bei der Abrechnungsprüfung hilft die systematische Fotodokumentation, Erlöseinbußen zu verhindern. \\ - Die Fotodokumentation erleichtert und beschleunigt die Abrechnungs- prüfung. \\ - Die Implementierung digitaler End- geräte mit entsprechender Software ist ein wichtiger Bestandteil des digi- talen Strukturwandels in Kliniken.}

\section{Korrespondenzadresse}

PD Dr. med.
Martin C. Jordan
Klinik und Poliklinik für
Unfall-, Hand-, Plastische und
Wiederherstellungschirurgie,
Universitätsklinikum
Würzburg
Oberdürrbacher Straße 6,
97080 Würzburg,
Deutschland
Jordan_M@ukw.de

Funding. Open Access funding provided by Projekt DEAL.

\section{Einhaltung ethischer Richtlinien}

Interessenkonflikt. M.C. Jordan, S. Jovic, F. Gilbert, A. Kunz, M. Ertl, U. Strobl, R.G. Jakubietz, M.G. Jakubietz, R.H. Meffert und K.F. Fuchs geben an, dass kein Interessenkonflikt besteht.

Für diesen Beitrag wurden von den Autoren keine Studien an Menschen oder Tieren durchgeführt. Für die aufgeführten Studien gelten die jeweils dort angegebenen ethischen Richtlinien. Für Bildmaterial oder anderweitige Angaben innerhalb des Manuskripts, über die Patienten zu identifizieren sind, liegt von innen und/oder ihren gesetzlichen Vertretern eine schriftliche Einwilligung vor.

Open Access. Dieser Artikel wird unter der Creative Commons Namensnennung 4.0 International Lizenz veröffentlicht, welche die Nutzung, Vervielfältigung, Bearbeitung, Verbreitung und Wiedergabe in jeglichem Medium und Format erlaubt, sofern Sie den/die ursprünglichen Autor(en) und die Quelle ordnungsgemäß nennen, einen Link zur Creative Commons Lizenz beifügen und angeben, ob Änderungen vorgenommen wurden.

Die in diesem Artikel enthaltenen Bilder und sonstiges Drittmaterial unterliegen ebenfalls der genannten Creative Commons Lizenz, sofern sich aus der Abbildungslegende nichts anderes ergibt. Sofern das betreffende Material nicht unter der genannten Creative Commons Lizenz steht und die betreffende Handlung nicht nach gesetzlichen Vorschriften erlaubt ist, ist für die oben aufgeführten Weiterverwendungen des $\mathrm{Ma}$ terials die Einwilligung des jeweiligen Rechteinhabers einzuholen.

Weitere Details zur Lizenz entnehmen Sie bitte der Lizenzinformation auf http://creativecommons.org/ licenses/by/4.0/deed.de.

\section{Literatur}

1. MDK Nordrhein (2019) Krankenhausrechnung: Prüfungen auf Rekordniveau. Pressemitteilung. https://www.mdk-nordrhein.de/nc/presse/ artikel/krankenhausrechnungen-pruefungenauf-rekordniveau/.Zugriffsdatum:20.04.2020

2. Verhoff MA, Kettner M, Laszik A, Ramsthaler F (2012) Digital photo documentation of forensically relevant injuries as part of the clinical first response protocol. Dtsch Arztebl Int 109:638-642. https:// doi.org/10.3238/arztebl.2012.0638

3. Solan MC, Calder JD, Gibbons CE, Ricketts DM (2001) Photographic wound documentation after open fracture. Injury 32:33-35. https://doi.org/10. 1016/s0020-1383(00)00124-8

4. Bloemen EM, Rosen T, Cline Schiroo JA, ClarkS, Mulcare MR, Stern ME, Mysliwiec R, Flomenbaum NE, Lachs MS, Hargarten S (2016) Photographing injuries in the acute care setting: development and evaluation of a standardized protocol for research, forensics, and clinical practice. Acad Emerg Med 23:653-659. https://doi.org/10.1111/acem.12955

5. Prantl L, Brandl D, Ceballos P (2017) A proposal for updated standards of photographic documentation in aesthetic medicine. Plast Reconstr Surg Glob Open 5:e1389. https://doi.org/10.1097/GOX. 0000000000001389

6. Kim SH, Sobez LM, Spiro JE, Curta A, Ceelen F, Kampmann E, Goepfert M, Bodensohn R, Mei- nel FG, Sommer WH, Sommer NN, Galie F (2020) Structured reporting has the potential to reduce reporting times of dual-energy $x$-ray absorptiometry exams. BMC Musculoskelet Disord 21:248. https://doi.org/10.1186/s12891-020-03200-w

7. Wallis LA, Fleming J, Hasselberg M, Laflamme L, Lundin J (2016) A smartphone app and cloudbased consultation system for burn injury emergency care. PLoS ONE 11:e147253. https:// doi.org/10.1371/journal.pone.0147253

8. Bouillion B (2016) S3-Leitlinie Polytrauma/ Schwerverletzten-Behandlung. Deutsche Gesellschaft für Unfallchirurgie. AWMF RegisterNr. 012/019:107

9. Li MK, Howard DP, King R (2019) "A picture tells a thousand words" smartphone-based secure clinical image transfer improves compliance in open fracture management. Injury 50:1284-1287. https://doi.org/10.1016/j.injury.2019.05.010

10. Augurzky B, Beivers A (2019) Digitalisierung und Investitionsfinanzierung. Krankenhaus-Report 2019. DOI. https://doi.org/10.1007/978-3-66258225-1_5(Kapitel 5)

11. Vogt $F$, Seidl F, Santarpino $G$, van Griensven $M$, Emmert M, Edenharter G, Pforringer D (2018) Healthcare IT utilization and penetration among physicians: novel IT solutions in healthcare-use and acceptance in hospitals. Eur Surg Res 59:100-113. https://doi.org/10.1159/000490241

12. Spitzer M (2019) Die Smartphone-Epidemie: Gefahr für Gesundheit, Bildung und Gesellschaft, 4. Aufl. Klett-Cotta, Stuttgart 
Hier steht eine Anzeige.

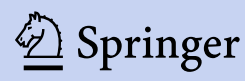

